International Journal of Algebra, Vol. 8, 2014, no. 19, 903 - 907

HIKARI Ltd, www.m-hikari.com

http://dx.doi.org/10.12988/ija.2014.212137

\title{
Morphisms Between the Groups of Semi Magic Squares and Real Numbers
}

\author{
K. S. Sreeranjini \\ Dept. of Mathematics \\ Amrita Vishwa Vidyapeetham \\ Coimbatore, India \\ V. Madhukar Mallayya \\ Dept. of Mathematics \\ MCET, Thiruvananthapuram, India
}

Copyright (c) 2014 K. S. Sreeranjini and V. Madhukar Mallayya. This is an open access article distributed under the Creative Commons Attribution License, which permits unrestricted use, distribution, and reproduction in any medium, provided the original work is properly cited.

\begin{abstract}
Homomorphisms and isomorphisms between the groups of semi magic squares and real numbers are discussed in this paper.

Mathematics Subject Classification: 15Axx
\end{abstract}

Keywords: Semi magic square, magic square, magic constant, group, homomorphism, isomorphism

\section{Introduction}

A magic square is a square array of numbers. If we add up the numbers in the square horizontally, vertically or diagonally, the sum remains the same. The constant sum is called magic constant or magic number. If the sum is a constant for the elements of rows and columns alone, that square is called a semi magic square. All magic squares are semi magic squares. We have seen many recreational aspects of magic squares and semi magic squares. But, 
apart from the usual recreational aspects, it is found that these squares possess advanced mathematical properties. Here we deal with some of such properties.

2. Notations and Mathematical Preliminaries (Basic ideas in this section are taken from [1] and [4].)

2.1 Magic Square: A magic square of order $n$ is a square array $\left[a_{i j}\right]$ of $n \times n$ numbers such that

$$
\begin{gathered}
\sum_{j=1}^{n} a_{i j}=k, \text { for } i=1,2,3, \ldots, n \ldots \ldots(1) \\
\sum_{j=1}^{n} a_{j i}=k, \text { for } i=1,2,3, \ldots, n \ldots \ldots(2) \\
\sum_{i=1}^{n} a_{i i}=k \text { and } \sum_{i=1}^{n} a_{i, n-i+1}=k \text { for } i=1,2,3, \ldots, n \ldots \ldots
\end{gathered}
$$

where $k$ is a constant, the above mentioned $a_{i j}$ 's, $a_{j i}$ 's are the row and column elements and $a_{i i}$ 's, $a_{i, n-i+1}$ 's are the left and right diagonal elements of the magic square respectively.

2.2 Magic Constant: The constant $k$ in the above definition is known as the magic constant or magic number. The magic constant of the magic square $A$ is denoted as $\rho(A)$.

- For example, the below given magic squares $A$ and $B$ are of order 3 with $\rho(A)=21$ and $\rho(B)=15$ respectively.

$$
A=\begin{array}{|c|c|c|}
\hline 4 & 9 & 8 \\
\hline 11 & 7 & 3 \\
\hline 6 & 5 & 10 \\
\hline
\end{array} \text { and } B=\begin{array}{|c|c|c|}
\hline 4 & 3 & 8 \\
\hline 9 & 5 & 1 \\
\hline 2 & 7 & 6 \\
\hline
\end{array}
$$

2.3 Semi magic square: In definition 2.1, if only conditions (1) and (2) are satisfied, then that square array is known as a semi magic square.

- For example, the below given array $C$ is a semi magic square of order 3 with $\rho(C)=30$.

$$
C=\begin{array}{|c|c|c|}
\hline 8 & 18 & 4 \\
\hline 16 & 2 & 12 \\
\hline 6 & 10 & 14 \\
\hline
\end{array}
$$

Here sum of elements of each row/column $=30$. Sum of the left diagonal elements $=8+2+14=24$ and sum of the right diagonal elements $=12$.

2.4: We use (i) $\mathbb{R}$ to denote the set of all real numbers. (ii) $S_{M}$ to denote the set of all $n^{\text {th }}$ order semi magic squares. (iii) $S_{a}$ to denote the set of all $n^{\text {th }}$ order semi magic squares of the form $\left[a_{i j}\right]$,

$$
a_{i j}=\left\{\begin{array}{cc}
a, & \text { for } i=j, i, j=1,2,3, \ldots, n \\
0, & \text { otherwise }
\end{array}\right.
$$


where $a \in \mathbb{R}$. (Here 0 is also a permitted value of $a . \rho\left(\left[a_{i j}\right]\right)=a$ ). If $A \in S_{a}$ with all the main diagonal entries equal to $a$, then it can be represented as $A \in S_{a}$ with $a_{i i}=a$. (iv) $f_{\rho}$ to denote the function $f: \mathbb{V} \rightarrow \mathbb{R}$, defined by $f(A)=\rho(A), \forall A \in \mathbb{V}$, where $\mathbb{V}$ is a subset of $S_{M}$. For example, $f_{\rho}: S_{M} \rightarrow \mathbb{R}$ means $f: S_{M} \rightarrow \mathbb{R}$ defined by $f(A)=\rho(A), \forall A \in S_{M}$.

\section{Propositions and Theorems}

Theorem 3.1: $<S_{M},+>$ is a group, where + denotes matrix addition.

Proof : [2], Theorem $3.1 \diamond$

Theorem 3.2: $\left\langle S_{a},+>\right.$ is a group, where + denotes matrix addition.

Proof: [3], Theorem $3.1 \diamond$

Proposition 3.1: The mapping $f_{\rho}: S_{M} \rightarrow \mathbb{R}$ is a group homomorphism.

Proof: Here we have to show that $f(A+B)=f(A)+f(B), \forall A, B \in S_{M}$. Let $A, B \in S_{M}$. Then

$f(A+B)=\rho(A+B)=\rho(A)+\rho(B)[$ by [2], Proposition 3.1 and result (ii) ] $=f(A)+f(B) \diamond$

Proposition 3.2: The kernel of the homomorphism in Proposition 3.1 is $k \operatorname{ker} f_{\rho}=\left\{A=\left[\frac{k}{n}-b_{i j}\right]\right.$, where $\left[b_{i j}\right]=B \in S_{M}$ with $\left.\rho(B)=k\right\}$. Let

Proof: We have $\operatorname{kerf}_{\rho}=\left\{A \in S_{M}, f(A)=0\right\}=\left\{A \in S_{M}, \rho(A)=0\right\}$.

$$
\varpi=\left\{A=\left[\frac{k}{n}-b_{i j}\right] \text {, where }\left[b_{i j}\right]=B \in S_{M} \text { with } \rho(B)=k\right\} .
$$

It is enough to show that $\varpi=k e r f_{\rho}$. Let $A \in \varpi$. Then $A$ will take the form $A=\left[\frac{k}{n}-b_{i j}\right]$, where $\left[b_{i j}\right]=B \in S_{M}$ with $\rho(B)=k \in \mathbb{R}$. Now,

$$
\begin{gathered}
\rho(A)=n \cdot \frac{k}{n}-\sum_{j=1}^{n} b_{i j}=k-k=0 \Rightarrow A \in \operatorname{kerf}_{\rho} \Rightarrow \varpi \subset \operatorname{ker} f_{\rho} \ldots . . \\
\left(\text { Since } B \in S_{M}, \sum_{j=1}^{n} b_{i j}=\rho(B)=k\right) .
\end{gathered}
$$

Let $C=\left[c_{i j}\right] \in \operatorname{kerf}_{\rho}$. Then $\rho(C)=0$. Now, $c_{i j}=\frac{0}{n}-\left(-c_{i j}\right)$ and so $C=\left[\frac{0}{n}-\left(-c_{i j}\right)\right]$. Here $\left[b_{i j}\right]=\left[-c_{i j}\right] \in S_{M}$ with $\rho\left(\left[b_{i j}\right]\right)=0$. Hence $C \in \varpi$ with $k=0$.

So $k e r f_{\rho} \subset \varpi \ldots \ldots(2)$. From (1) and (2) $\varpi=k e r f_{\rho} \diamond$

Remark: The mapping $f_{\rho}: S_{M} \rightarrow \mathbb{R}$ is a group homomorphism and is not a group isomorphism, since $\operatorname{ker} f_{\rho} \neq 0$.

Proposition 3.3: The mapping $f_{\rho}: S_{a} \rightarrow \mathbb{R}$ is a group homomorphism.

Proof : Let $A=\left[a_{i j}\right], B=\left[b_{i j}\right] \in S_{a}$ with $a_{i i}=a$ and $b_{i i}=b$. Then, $\rho(A)=a, \rho(B)=b$ and $A+B=\left[a_{i j}+b_{i j}\right]$ 
where

$$
a_{i j}+b_{i j}=\left\{\begin{array}{cc}
a+b, & \text { for } i=j, i, j=1,2,3, \ldots, n \\
0, & \text { otherwise }
\end{array}\right.
$$

$A+B \in S_{a}$ with $\rho(A+B)=a+b$. Now,

$$
f(A+B)=\rho(A+B)=a+b=\rho(A)+\rho(B)=f(A)+f(B) \diamond
$$

Proposition 3.4: The mapping $f_{\rho}: S_{a} \rightarrow \mathbb{R}$ is a group isomorphism.

Proof : By Proposition 3.3, $f_{\rho}: S_{a} \rightarrow \mathbb{R}$ is a group homomorphism. So it is enough to show that $f_{\rho}: S_{a} \rightarrow \mathbb{R}$ is a one - one onto mapping.

(i) $f_{\rho}$ is one - one

Let $A=\left[a_{i j}\right]$ and $B=\left[b_{i j}\right] \in S_{a}$ with $a_{i i}=a$ and $b_{i i}=b$. Here $\rho(A)=a$ and $\rho(B)=b$. Now,

$$
\begin{gathered}
f(A)=f(B) \Rightarrow \rho(A)=\rho(B) \\
\Rightarrow a=b \Rightarrow a_{i i}=b_{i i} \Rightarrow\left[a_{i j}\right]=\left[b_{i j}\right] \\
\left(\text { Since, } A=\left[a_{i j}\right] \text { and } B=\left[b_{i j}\right] \in S_{a}\right) \\
\text { i.e., } A=B
\end{gathered}
$$

(ii) $f_{\rho}$ is onto

$\forall a \in \mathbb{R}, \exists A=\left[a_{i j}\right] \in S_{a}$ with $a_{i i}=a$ and $f(A)=\rho(A)=a \diamond$

\section{Conclusion}

We have seen that the mapping $f_{\rho}: S_{M} \rightarrow \mathbb{R}$ is a group homomorphism and not a group isomorphism. We also found the kernel of the homomorphism. The mapping $f_{\rho}: S_{a} \rightarrow \mathbb{R}$ is a group homomorphism as well as a group isomorphism.

\section{References}

[1] W.S. Andrews, Magic Squares and Cubes - second edition, Dover Publications, Inc. 180 Varick Street, New York 14, N.Y. (1960).

[2] Sreeranjini K.S, V.Madhukar Mallayya, Some Special Properties of Semi Magic Squares, International Journal of Advance in Mathematics and Mathematical Sciences, July - Dec 2012, Academic Research Journals, India.

[3] Sreeranjini K.S, V. Madhukar Mallayya, Semi Magic Squares as a Field, International Journal of Algebra, Vol. 6, 2012, no. 26, 1249 - 1256.

[4] A. R. Vasishtha and A. K. Vasishtha, Modern Algebra - fifth edition, Krishna Prakashan Media (P) Ltd., H.O. - 11, Shivaji Road, Meerut - 250001 
(U.P.), 2006.

Received: December 15, 2012; Published: December 21, 2014 